\title{
A Study on the Use of Compound and Extracted Models in the High Frequency Electromagnetic Exposure Assessment
}

\author{
Mario Cvetković, ${ }^{1}$ Hrvoje Dodig, ${ }^{2}$ and Dragan Poljak ${ }^{1}$ \\ ${ }^{1}$ Faculty of Electrical Engineering, Mechanical Engineering and Naval Architecture, University of Split, \\ R. Boškovića 32, 21000 Split, Croatia \\ ${ }^{2}$ Faculty of Maritime Studies, University of Split, R. Boškovića 37, 21000 Split, Croatia \\ Correspondence should be addressed to Mario Cvetković; mcvetkov@fesb.hr
}

Received 12 July 2017; Accepted 12 October 2017; Published 26 November 2017

Academic Editor: Peter Dabnichki

Copyright (C) 2017 Mario Cvetković et al. This is an open access article distributed under the Creative Commons Attribution License, which permits unrestricted use, distribution, and reproduction in any medium, provided the original work is properly cited.

\begin{abstract}
The paper presents the numerical results for the induced electric field in the various models of the human eye and the head. The comparison between the extracted or the single organ models and the compound organ models placed inside realistic head models obtained from the magnetic resonance imaging scans is presented. The numerical results for several frequencies and polarizations of the incident electromagnetic (EM) plane wave are obtained using the hybrid finite element method/boundary element method (FEM/BEM) formulation and the surface integral equation (SIE) based formulation featuring the use of method of moments, respectively. Although some previous analysis showed the similar distribution of the induced electric field along the pupillary axis obtained in both eye models, this study showed this not to be the case in general. The analysis showed that the compound eye model is much more suitable when taking into account the polarization of the incident EM wave. The numerical results for the brain models showed much better agreement in the maximum values and distributions of the induced surface field between detailed models, while homogeneous brain model showed better agreement with the compound model in the distribution along selected sagittal axis points. The analysis could provide some helpful insights when carrying out the dosimetric analysis of the human eye and the head/brain exposed to high frequency EM radiation.
\end{abstract}

\section{Introduction}

The electromagnetic (EM) fields generated by the various wireless communication equipment such as mobile phones and base station antennas have increased the concern among the general population related to the possible harmful effects. As the established biological effect of high frequency (HF) electromagnetic fields is tissue heating, the assessment of this HF exposure is based on determining the specific absorption rate (SAR) that is related to the electric field induced in the tissue. The HF exposure assessment is particularly important in the case of human eye and brain since experimental measurement in healthy humans is very difficult if not impossible. The solution to this is the use of the computational models and the related numerical solutions as a tool for assessment of HF exposure [1-3].

The computational models employed for this particular type of assessment can be classified as realistic models of the human body (or particular organs of interest) based on the magnetic resonance imaging (MRI) (e.g. [4]) or the simplified models, computationally much less demanding but failing to provide accurate results in most of the exposure scenarios [5].

The detailed models of the complete human body are nowadays readily available (e.g., $[6,7])$; however, the detailed human body model puts the significant burden in the computational model preparation at the same time putting strain on the available computational resources. In addition to this, there are cases when only the particular organ or body parts are of research interest, as when the initial assessment is considered.

The selection between the simplified single organ model and the more detailed and complete body model is not simple nor straightforward. This paper is an extension of recent study [8] on the comparison of the results between the compound and the extracted eye models, respectively, exposed to $1 \mathrm{GHz}$ electromagnetic plane wave at single polarization. This paper 


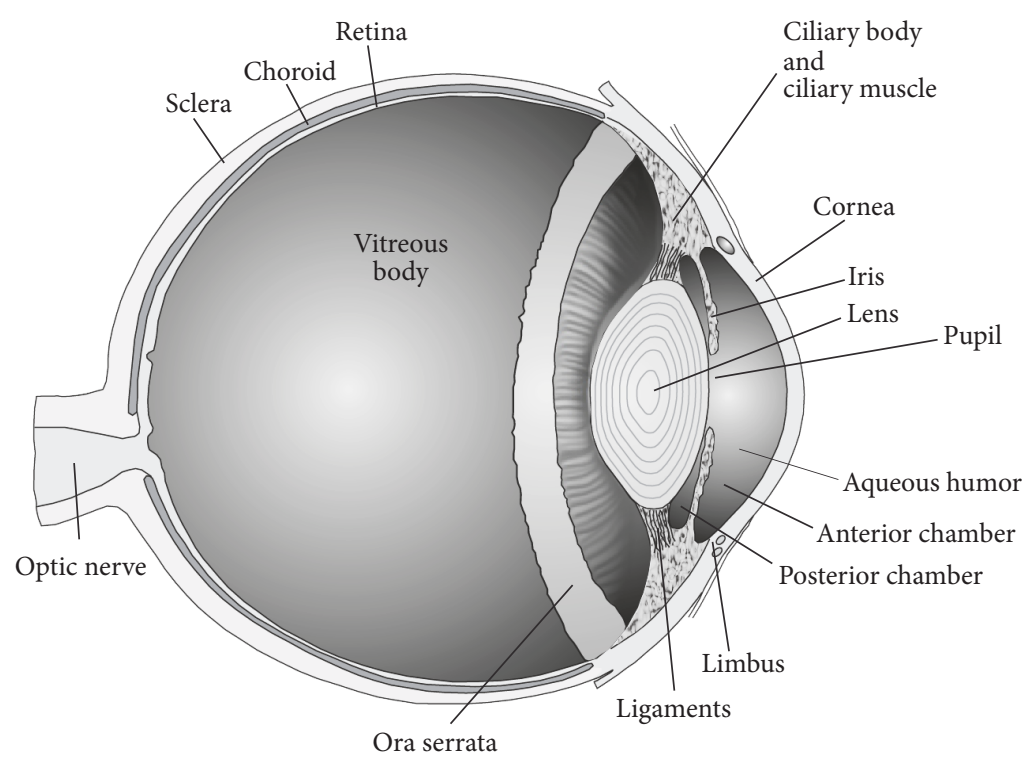

FIGURE 1: Sagittal cross-section of the human eye depicting various tissues.

deals with high frequency EM exposure in case of the human eye and human head/brain exposed to several frequencies and polarizations. The single organ models of the eye and the brain, termed here the extracted or the single models, and those models incorporated in the detailed human head model, termed the compound models, are used in the numerical assessment of the induced electric field due to high frequency electromagnetic radiation.

The paper is organized as follows. The first part gives a description of the extracted and the compound eye and brain models, respectively. Following this, the description of the hybrid finite element method/boundary element method (FEM/BEM) approach [9] and the surface integral equation (SIE) approach [10], respectively, are given. The corresponding numerical solutions are reported as well. The numerical results for the induced electric field in two eye models exposed to $1 \mathrm{GHz}$ and $1800 \mathrm{MHz}$ EM plane wave are given in the following section. The same section features another set of results related to the induced field in three human brain/head models exposed to $900 \mathrm{MHz}$ and $1800 \mathrm{MHz}$ EM plane wave. The discussion of the results and the conclusion are given in the final part.

\section{Materials and Methods}

2.1. Extracted (Single) Eye Model. The human eye is a delicate organ consisting of many fine parts, each performing various important functions. In order to account for such a small but important subtleties, hence, a detailed model of the eye is very important. The sagittal cross-section of the human eye depicting its parts is shown in Figure 1.

Magnetic resonance imaging (MRI) can very accurately capture details of the human anatomy; however, the spatial resolution of MRI is not sufficient to capture the fine geometrical details of the human eye. According to some recent studies, ultra-high resolution $7 \mathrm{~T}$ MRI techniques were able to capture spatial anatomical data with isotropic resolutions of $0.6 \mathrm{~mm}$ and $0.7 \mathrm{~mm}$ [11] while [12] reported spatial resolutions of $0.5 \times 0.5 \times 0.6 \mathrm{~mm}^{3}$. To the best of the authors knowledge, the most accurate MRI scans were performed using the $9.4 \mathrm{~T}$ magnet [13] achieving voxel volumes of about $0.13 \times 0.13 \times 0.8 \mathrm{~mm}^{3}$. However, in order to capture the fine details of the eye tissues such as choroid, retina, and iris, a much finer resolution is required. It is estimated that in order to have at least several layers of MRI voxels in the retina, one would need spatial MRI resolution of about $30 \times 30 \times 30 \mu \mathrm{m}^{3}$ or less, or the resulting boundary surfaces between tissues would have become staircased.

Therefore, the detailed geometrical model of the human eye, named here as the extracted (single) eye model, has been developed from the available MRI scans as well as from various medical measurements data. Modeled tissues from the extracted and the compound eye models are shown in Figure 2.

The extracted eye model consists of 16 tissues, whose parameters are given in Table 1 . The frequency dependent dielectric parameters (the electrical conductivity $\sigma$ and the relative permittivity $\varepsilon$ ) are modeled using the 4-Cole-Cole method [14]. Table 1 gives also the tissue mass density $\rho$.

The crystalline lens is modeled using five layers with varying relative permittivity, according to the Gradient Refraction Index (GRIN) model $[15,16]$.

The boundary surface of the eye model is discretized using 7.986 triangular elements, while interior domain of the eye is discretized using 415.429 tetrahedral elements.

2.2. Compound Eye and Head Model. The extracted eye model developed in the previous section is incorporated in the full model of the human head composed of various head tissues as shown in Figure 3(c). This model is named the compound eye model. 


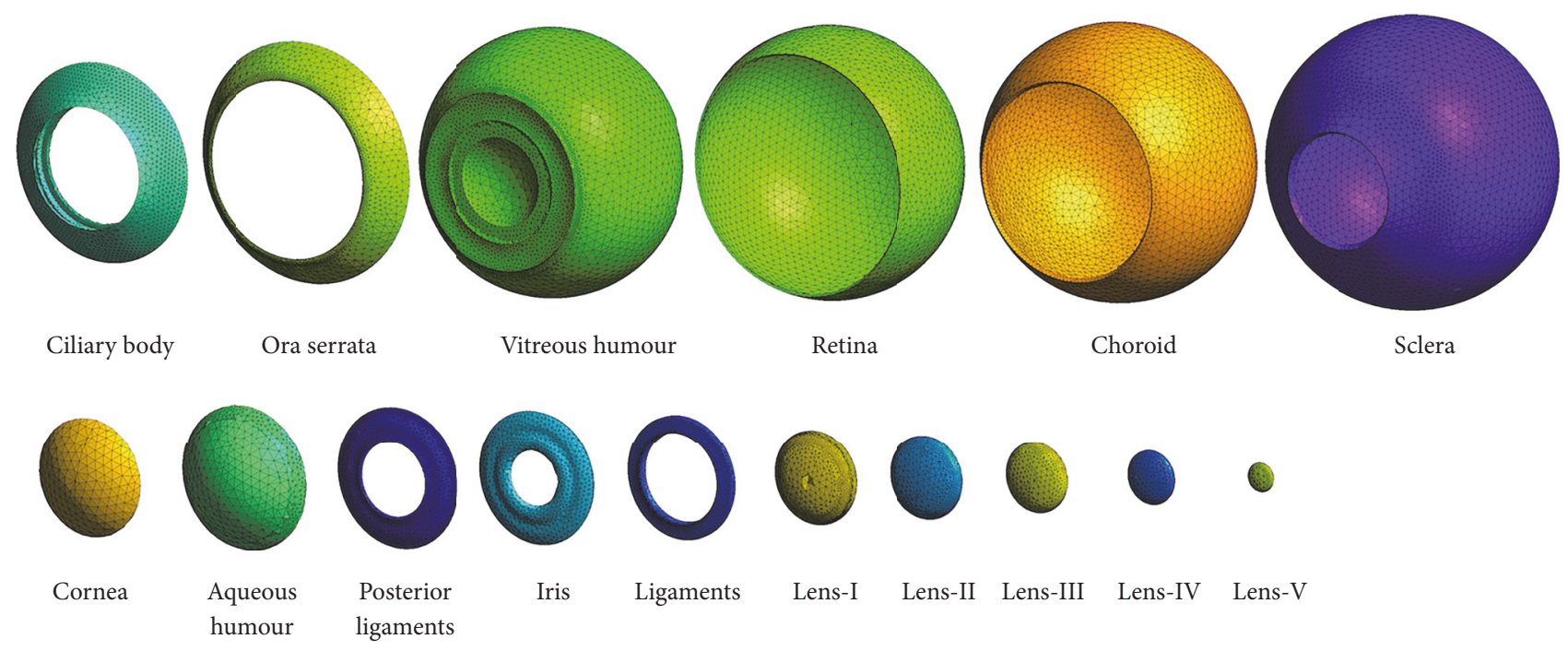

FIGURE 2: Modeled tissues from the extracted and the compound eye models.

TABLE 1: Tissue parameters used in eye and head models.

\begin{tabular}{lccccc}
\hline \multirow{2}{*}{ Tissue } & \multicolumn{3}{c}{$900 \mathrm{MHz}$} & \multicolumn{3}{c}{$1800 \mathrm{MHz}$} & \multirow{2}{*}{$\left(\mathrm{g} / \mathrm{m}^{3}\right)$} \\
& $\sigma(\mathrm{S} / \mathrm{m})$ & $\varepsilon(-)$ & $\sigma(\mathrm{S} / \mathrm{m})$ & $\varepsilon(-)$ & \\
\hline Brainstem & 0.622 & 38.577 & 0.915 & 37.011 & 1043 \\
Cerebellum & 1.308 & 48.858 & 1.709 & 46.114 & 1039 \\
Head skin & 0.899 & 40.936 & 1.185 & 38.872 & 1050 \\
Liquor & 1.667 & 68.875 & 2.032 & 68.573 & 1035 \\
Skull & 0.364 & 20.584 & 0.588 & 19.343 & 1900 \\
Mandible & 0.364 & 20.584 & 0.588 & 19.343 & 1900 \\
Grey matter & 0.985 & 52.282 & 1.391 & 50.079 & 1039 \\
\hline Anterior chamber & 1.667 & 68.875 & 2.032 & 68.573 & 1003 \\
Choroid & 0.729 & 44.561 & 1.066 & 43.343 & 1000 \\
Ciliary body & 0.978 & 54.811 & 1.341 & 53.549 & 1040 \\
Cornea & 1.438 & 54.835 & 1.858 & 52.768 & 1076 \\
Iris & 0.978 & 54.811 & 1.341 & 53.549 & 1040 \\
Ligaments & 0.760 & 45.634 & 1.201 & 44.252 & 1000 \\
Ora serrata & 0.882 & 45.711 & 1.232 & 43.850 & 1000 \\
Posterior chamber & 1.667 & 68.875 & 2.032 & 68.573 & 1000 \\
Retina & 1.206 & 55.017 & 1.602 & 53.568 & 1039 \\
Sclera & 1.206 & 55.017 & 1.602 & 53.568 & 1076 \\
Vitreous body & 1.667 & 68.875 & 2.032 & 68.573 & 1009 \\
\hline Lens-I & 0.824 & 46.399 & 1.147 & 45.353 & 1100 \\
Lens-II & 0.824 & 47.011 & 1.147 & 45.925 & 1100 \\
Lens-III & 0.824 & 47.694 & 1.147 & 46.221 & 1100 \\
Lens-IV & 0.824 & 48.383 & 1.147 & 46.883 & 1100 \\
Lens-V & 0.824 & 49.076 & 1.147 & 47.554 & 1100 \\
\hline
\end{tabular}

The detailed model of the human head was constructed from the MRI of a 24-year-old male [17]. The current implementation of the model features 7 tissues in addition to 16 ocular tissues from the extracted eye model. The head tissue parameters are also modeled using the 4-Cole-Cole method [14] and are given in Table 1. The boundary surface of the complete head model is discretized using 5.934 triangular elements, while region inside the head is discretized using 4.073.250 tetrahedral elements.

2.3. Homogeneous Brain Model. As a very simple representation of the human brain, shown in Figure 3(a), the homogenized realistic-shaped brain reported in [10] was used. To facilitate the preparation of otherwise a very complex organ, the freely available model from Google SketchUp was scaled to adult average brain using dimensions of length $167 \mathrm{~mm}$, width $140 \mathrm{~mm}$, height $93 \mathrm{~mm}$, and volume of $1400 \mathrm{~cm}^{3}$ [18]. The surface of the model was discretized using the triangular elements, enabling the use of Rao-Wilton-Glisson (RWG) basis functions specially developed for triangular patches.

Frequency dependent parameters of the homogeneous models are taken from [10]. In addition, linear and isotropic behavior is assumed for the electrical properties of tissues.

It should be noted that the employed brain geometry is an extremely simplified model, as the brain surface was radically smoothed thus missing complex folding structures of gyri and sulci, in addition to consisting of a single, homogeneous structure. Nonetheless, it is important to emphasize that this homogeneous model could still be useful as in the initial assessment comparison [19].

Although it is more realistic than the sphere, it lacks the detailed cortical structures and inhomogeneity (grey/white matter, ventricles, etc.). In order to overcome this limitation, the future work should therefore include comparisons on detailed anatomically correct head model, featuring complex material maps and shapes. Nonetheless, regarding the use of the homogeneous model it is important to emphasize that it is reasonable to start comparing different numerical techniques using simple models thus opening the subject.

2.4. Three-Compartment Head Model. The next logical step in the development of a more realistic brain model would be to place it inside the various surrounding tissues. The most realistic head model routinely used in experimental magnetoencephalography (MEG) is the so-called three-shell 


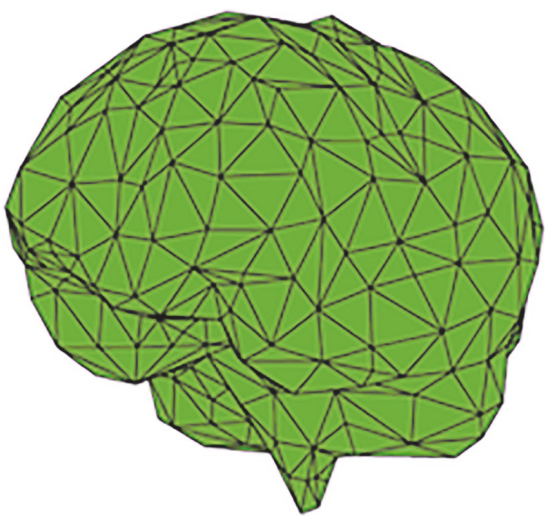

(a)

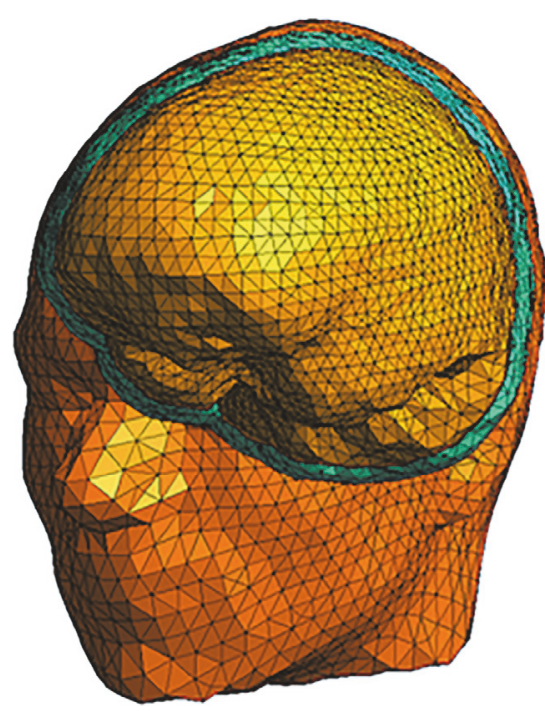

(b)

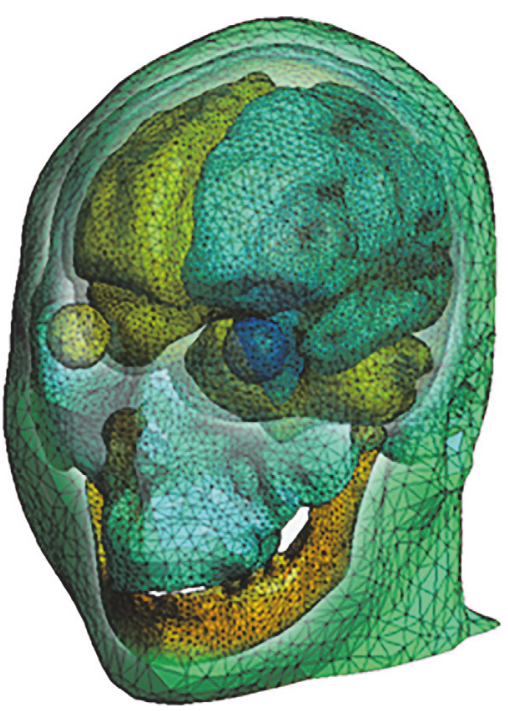

(c)

FIGURE 3: Models for the brain comparison: (a) homogeneous brain model, (b) three-compartment head model, and (c) compound model. Overlay on two latter models is showing various head tissues surrounding the brain.

TABLE 2: Tissue parameters used in the three-compartment model.

\begin{tabular}{lccccc}
\hline \multirow{2}{*}{ Tissue } & \multicolumn{2}{c}{$900 \mathrm{MHz}$} & \multicolumn{2}{c}{$1800 \mathrm{MHz}$} & \multirow{2}{*}{$\left(\mathrm{g} / \mathrm{m}^{3}\right)$} \\
& $\sigma(\mathrm{S} / \mathrm{m})$ & $\varepsilon(-)$ & $\sigma(\mathrm{S} / \mathrm{m})$ & $\varepsilon(-)$ & \\
\hline Scalp & 0.899 & 40.936 & 1.185 & 38.872 & 1050 \\
Brain & 0.985 & 52.282 & 1.391 & 50.079 & 1039 \\
Skull & 0.364 & 20.584 & 0.588 & 19.343 & 1900 \\
\hline
\end{tabular}

or the three-compartment model [20], consisting of homogeneous compartments of brain, skull, and scalp, as depicted in Figure 3(b). This model is freely available and can be easily implemented in the BEM analysis [20]. The parameters of the three-shell model are given in Table 2 .

2.5. Hybrid FEM/BEM Method. Electromagnetic wave incident on the human eye or head can be treated as unbounded scattering problem. Using the Stratton-Chu integral expression, the time harmonic electric field in the domain exterior to the head is expressed by the following boundary integral equation $[9,21]$ :

$$
\begin{aligned}
\alpha \vec{E}_{\mathrm{ext}}^{\prime}= & \vec{E}_{\mathrm{inc}}^{\prime}+\oint_{\partial V} \hat{n} \times\left(\nabla \times \vec{E}_{\mathrm{ext}}\right) G d S \\
& +\oint_{\partial V}\left[\left(\widehat{n} \times \vec{E}_{\mathrm{ext}}\right) \times \nabla G+\left(\widehat{n} \cdot \vec{E}_{\mathrm{ext}}\right) \nabla G\right] d S,
\end{aligned}
$$

where $\widehat{n}$ is an outer normal to surface $\partial V$ bounding the volume $V$ and $\alpha$ is the solid angle subtended at the observation point. The total and the incident electric field are denoted by $\vec{E}_{\text {ext }}$ and $\vec{E}_{\text {inc }}$, respectively, and $G$ is the free space Green's function given by

$$
G=G\left(\vec{r}, \vec{r}^{\prime}\right)=\frac{e^{-j k R}}{4 \pi R} ; \quad R=\left|\vec{r}-\vec{r}^{\prime}\right|,
$$

where $R$ is the distance from the observation point $\vec{r}$ to the source point $\vec{r}^{\prime}$ and $k$ denotes the wave number.

Performing some manipulations, (1) can be specified in terms of tangential components of electric field $\vec{E}$ and magnetic field $\vec{H}$ on the boundary surface $\partial V[9]$ :

$$
\begin{aligned}
& \alpha \vec{E}_{\mathrm{ext}}^{\prime}=\vec{E}_{\mathrm{inc}}^{\prime}-j \omega \mu \oint_{\partial V} \widehat{n} \times \vec{H}_{\mathrm{ext}} G d S \\
& \quad+\oint_{\partial V}\left[\left(\widehat{n} \times \vec{E}_{\mathrm{ext}}\right) \times \nabla G-\frac{1}{\sigma+j \omega \mu} \nabla_{s}\right. \\
& \left.\quad \cdot\left(\widehat{n} \times \vec{H}_{\mathrm{ext}}\right) \nabla G\right] d S .
\end{aligned}
$$

The form of (3) is pertinent for coupling to the governing differential equations of the interior inhomogeneous domain, given by [9]:

$$
\nabla \times\left(\frac{j}{\omega \mu} \nabla \times \vec{E}_{\mathrm{int}}\right)-(\sigma+j \omega \varepsilon) \vec{E}_{\mathrm{int}}=0 .
$$

The fields $\vec{E}$ and $\vec{H}$ are approximated using the edge elements [22] preserving the tangential continuity of the fields on the boundary

$$
\begin{aligned}
& \vec{E}=\sum_{i=1}^{n} \delta_{i} \vec{w}_{i} e_{i}, \\
& \vec{H}=\sum_{i=1}^{n} \delta_{i} \vec{w}_{i} h_{i} .
\end{aligned}
$$

The unknown coefficients $e_{i}$ and $h_{i}$, respectively, associated with each edge of the model, are determined from the global system of equations while the coefficient $\delta_{i}= \pm 1$ is equal to 1 depending on whether the direction of local edge coincides with the chosen global edge direction. 
Vector base function $w_{k}$ is given by [23] $\vec{w}_{k}=N_{i} \nabla N_{j}-$ $N_{j} \nabla N_{i}$, where $N_{i}$ and $N_{j}$ are the first order barycentric shape functions.

After the weighted residual approach is applied to (4), followed by the dot product of (4) with test function $w_{i}$, and using the Galerkin-Bubnov procedure, it can be written that

$$
\int_{V} \delta_{i} \vec{w}_{i} \cdot\left[\nabla \times\left(\frac{j}{\omega \mu} \nabla \times \vec{E}_{\text {int }}\right)-(\sigma+j \omega \varepsilon) \vec{E}_{\text {int }}\right] d V=0 .
$$

After applying some standard vector identities, followed by the divergence theorem, the weak form is obtained:

$$
\begin{aligned}
\int_{V} & {\left[\frac{j}{\omega \mu} \nabla \times \delta_{i} \vec{w}_{i} \cdot \vec{E}_{\mathrm{int}}-(\sigma+j \omega \varepsilon) \delta_{i} \vec{w}_{i} \cdot \vec{E}_{\mathrm{int}}\right] d V } \\
= & \oint_{\partial V} \overrightarrow{d S} \cdot \delta_{i} \vec{w}_{i} \times \vec{H}_{\mathrm{int}} .
\end{aligned}
$$

The FEM/BEM coupling can now be employed by using the continuity of the tangential components of electric and magnetic fields across the surface $\partial V$. This leads to $\widehat{n} \times \vec{E}_{\text {int }}=$ $\widehat{n} \times \vec{E}_{\text {ext }}$ and $\widehat{n} \times \vec{H}_{\text {int }}=\hat{n} \times \vec{H}_{\text {ext }}$. Thus, it can be written that

$$
\oint_{\partial V} \overrightarrow{d S}^{\prime} \cdot \delta_{i} \vec{w}_{i} \times \vec{E}_{\mathrm{int}}^{\prime}=\oint_{\partial V} \overrightarrow{d S}^{\prime} \cdot \delta_{i} \vec{w}_{i} \times \vec{E}_{\mathrm{ext}}^{\prime}
$$

After substituting $\vec{E}_{\text {ext }}$ and $\vec{H}_{\text {ext }}$ in (3) with $\vec{E}_{\text {int }}$ and $\vec{H}_{\text {int }}$, respectively, and after inserting (3) into (8), the following double surface integral is obtained:

$$
\begin{aligned}
& \oint_{\partial V} \overrightarrow{d S}^{\prime} \cdot \delta_{i} \vec{w}_{i} \times \alpha \vec{E}_{\mathrm{ext}}^{\prime}=\oint_{\partial V} \overrightarrow{d S}^{\prime} \cdot \delta_{i} \vec{w}_{i} \times \vec{E}_{\mathrm{inc}}^{\prime}-j \omega \mu \\
& \cdot \oint_{\partial V} \overrightarrow{d S}^{\prime} \cdot \delta_{i} \vec{w}_{i} \\
& \times \oint_{\partial V} \hat{n} \times \vec{H}_{\mathrm{int}} G d S+\oint_{\partial V} \overrightarrow{d S}^{\prime} \cdot \delta_{i} \vec{w}_{i} \\
& \times \oint_{\partial V}\left(\hat{n} \times \vec{E}_{\mathrm{int}}\right) \times \nabla G d S-\frac{1}{\omega+j \omega \mu} \\
& \cdot \oint_{\partial V} \overrightarrow{d S}^{\prime} \cdot \delta_{i} \vec{w}_{i} \times \oint_{\partial V} \nabla_{s} \cdot\left(\hat{n} \times \vec{H}_{\mathrm{ext}}\right) \nabla G d S .
\end{aligned}
$$

Inserting (5) into (9) results in the system of equations related to the edges at the boundary surface of the problem:

$$
\begin{aligned}
& {\left[E_{\text {bem }}\right]\left\{e_{\text {bem }}\right\}=\left\{e_{\text {inc }}\right\}+\left[H_{\text {bem }}\right]\left\{h_{\text {bem }}\right\}} \\
& {\left[E_{\text {fem }}\right]\left\{e_{\text {fem }}\right\}=\left[H_{\text {fem }}\right]\left\{h_{\text {fem }}\right\},}
\end{aligned}
$$

where $e_{\text {bem }}$ and $h_{\text {bem }}$ are unknown coefficients associated with boundary surface of the scattering problem, $e_{\text {inc }}$ are known coefficients calculated from the incident field, and matrices $\left[E_{\text {bem }}\right]$ and $\left[H_{\text {bem }}\right]$ arise from boundary integral equation (9), while matrices $\left[E_{\text {fem }}\right]$ and $\left[H_{\text {fem }}\right]$ stem from FEM (7).

After having discretized the interior domain into finite set of tetrahedrons, and using the expansion given by (5), followed by the Galerkin procedure, the finite element matrices could be written. Thus, the element matrix $[K]^{e}$ of the first term from (7), after some work, can be written as

$$
\begin{aligned}
\frac{1}{k_{B}} \sum_{i=1}^{6} \sum_{j=1}^{6}\left(\nabla \times \vec{w}_{i} \delta_{i}\right) \cdot\left(\nabla \times \vec{w}_{j} \delta_{j}\right)\left\{\mathbf{a}_{\mathbf{i}}\right\} d V \\
=\frac{V_{\Delta}}{k_{B}}\left[\begin{array}{llllll}
k_{11} & k_{12} & k_{13} & k_{14} & k_{15} & k_{16} \\
k_{21} & k_{22} & k_{23} & k_{24} & k_{25} & k_{26} \\
k_{31} & k_{32} & k_{33} & k_{34} & k_{35} & k_{36} \\
k_{41} & k_{42} & k_{43} & k_{44} & k_{45} & k_{46} \\
k_{51} & k_{52} & k_{53} & k_{54} & k_{55} & k_{56} \\
k_{61} & k_{62} & k_{63} & k_{64} & k_{65} & k_{66}
\end{array}\right] \cdot\left\{\begin{array}{l}
a_{1} \\
a_{2} \\
a_{3} \\
a_{4} \\
a_{5} \\
a_{6}
\end{array}\right\},
\end{aligned}
$$

where $V_{\Delta}$ denotes the volume of the finite element tetrahedron and the coefficients $k_{i j}$ can be calculated from

$$
k_{i j}=\delta_{i} \delta_{j}\left(\nabla \times \vec{w}_{i}\right) \cdot\left(\nabla \times \vec{w}_{j}\right) .
$$

The element matrix $[M]^{e}$ of the second term from (7) can be written as

$$
k_{A} \sum_{i=1}^{6} \sum_{j=1}^{6} \int_{\Delta} \delta_{i} \delta_{j} \vec{w}_{i} \cdot \vec{w}_{j}\left\{\mathbf{a}_{\mathbf{i}}\right\} d V,
$$

where the dot product for edges $m$ and $n$ can be expanded as

$$
\begin{aligned}
\overrightarrow{w_{m}} \cdot \overrightarrow{w_{n}}= & {\left[N_{i}^{(m)} \nabla N_{j}(m)-N_{j}^{(m)} \nabla N_{i}(m)\right] } \\
& \cdot\left[N_{i}^{(n)} \nabla N_{j}(n)-N_{j}^{(n)} \nabla N_{i}(n)\right] \\
= & N_{i}^{(m)} N_{i}^{(n)} \nabla N_{j}^{(m)} \nabla N_{j}^{(n)} \\
& -N_{i}^{(m)} N_{j}^{(n)} \nabla N_{j}^{(m)} \nabla N_{i}^{(n)} \\
& -N_{j}^{(m)} N_{i}^{(n)} \nabla N_{i}^{(m)} \nabla N_{j}^{(n)} \\
& +N_{j}^{(m)} N_{j}^{(n)} \nabla N_{i}^{(m)} \nabla N_{i}^{(n)} .
\end{aligned}
$$

The procedure for calculating the volume integrals from (14) can be found elsewhere [24].

Finally, the element matrix $[D]^{e}$ of the right hand side from (7) can be written as

$$
\sum_{i=1}^{3} \sum_{j=1}^{3} \int_{\Delta} \overrightarrow{d S} \cdot \vec{w}_{i} \delta_{i} \times \vec{w}_{j} \delta_{j}\left\{\mathbf{a}_{\mathbf{i}}\right\},
$$

where the symbol $\Delta$ denotes the integration over triangle, and the vector product for edges $m$ and $n$ can be expanded as

$$
\begin{aligned}
\overrightarrow{w_{m}} \times \overrightarrow{w_{n}}= & N_{i}^{(m)} N_{i}^{(n)} \nabla N_{j}^{(m)} \times \nabla N_{j}^{(n)} \\
& -N_{i}^{(m)} N_{j}^{(n)} \nabla N_{j}^{(m)} \times \nabla N_{i}^{(n)} \\
& -N_{j}^{(m)} N_{i}^{(n)} \nabla N_{i}^{(m)} \times \nabla N_{j}^{(n)} \\
& +N_{j}^{(m)} N_{j}^{(n)} \nabla N_{i}^{(m)} \times \nabla N_{i}^{(n)} .
\end{aligned}
$$

The procedure for calculating the surface integrals from (16) can be found in [24]. 
2.6. SIE/MoM Approach for the Homogeneous Model. The human brain exposure can be estimated by means of coupled surface integral equations (SIE) [25, 26]:

$$
\begin{gathered}
j \omega \mu_{n} \iint_{S} \vec{J}\left(\vec{r}^{\prime}\right) G_{n}\left(\vec{r}, \vec{r}^{\prime}\right) d S^{\prime}-\frac{j}{\omega \varepsilon_{n}} \\
\cdot \iint_{S} \nabla_{S}^{\prime} \cdot \vec{J}\left(\vec{r}^{\prime}\right) \nabla G_{n}\left(\vec{r}, \vec{r}^{\prime}\right) d S^{\prime} \\
+\iint_{S} \vec{M}\left(\vec{r}^{\prime}\right) \times \nabla^{\prime} G_{n}\left(\vec{r}, \vec{r}^{\prime}\right) d S^{\prime} \\
= \begin{cases}\vec{E}^{\text {inc }}, & n=1 \\
0, & n=2,\end{cases}
\end{gathered}
$$

where $\vec{J}$ and $\vec{M}$ are equivalent electric and magnetic current density, respectively, $k_{n}$ is the wave number of a medium $n(n=1,2)$, while $G_{n}$ is the interior/exterior Green function for the homogeneous medium $[25,26]$ :

$$
G_{n}\left(\vec{r}, \vec{r}^{\prime}\right)=\frac{e^{-j k_{n} R}}{4 \pi R} ; \quad R=\left|\vec{r}-\vec{r}^{\prime}\right| .
$$

And $R$ is the distance from the source to observation point.

Set of integral equations (17) is solved by means of method of moments (MoM) procedure reported in [26]. The equivalent electric and magnetic currents $\vec{J}$ and $\vec{M}$ in (17) are expressed in terms of a linear combination of basis functions $\vec{f}_{n}$ and $\vec{g}_{n}$, respectively.

$$
\begin{aligned}
& \vec{J}(\vec{r})=\sum_{n=1}^{N} J_{n} \vec{f}_{n}(\vec{r}), \\
& \vec{M}(\vec{r})=\sum_{n=1}^{N} M_{n} \vec{g}_{n}(\vec{r}),
\end{aligned}
$$

where $J_{n}$ and $M_{n}$ are unknown coefficients, while $N$ is the total number of triangular elements.

Applying the weighted residual approach, that is, multiplying (17) by the set of a test functions $\vec{f}_{m}$ and integrating over the surface $S$, after performing some mathematical manipulations, it follows that

$$
\begin{aligned}
& j \omega \mu_{i} \sum_{n=1}^{N} J_{n} \iint_{S} \vec{f}_{m}(\vec{r}) \\
& \quad \cdot \iint_{S^{\prime}} \vec{f}_{n}\left(\vec{r}^{\prime}\right) G_{i} d S^{\prime} d S+\frac{j}{\omega \varepsilon_{i}} \sum_{n=1}^{N} J_{n} \iint_{S} \nabla_{S} \\
& \quad \cdot \vec{f}_{m}(\vec{r}) \iint_{S^{\prime}} \nabla_{S}^{\prime} \cdot \vec{f}_{n}\left(\vec{r}^{\prime}\right) G_{i} d S^{\prime} d S \\
& \quad \pm \sum_{n=1}^{N} M_{n} \iint_{S} \vec{f}_{m}(\vec{r}) \cdot\left[\widehat{n} \times \vec{g}_{n}\left(\vec{r}^{\prime}\right)\right] d S \\
& \quad+\sum_{n=1}^{N} M_{n} \iint_{S} \vec{f}_{m}(\vec{r})
\end{aligned}
$$

$$
\begin{gathered}
\cdot \iint_{S^{\prime}} \vec{g}_{n}\left(\vec{r}^{\prime}\right) \times \nabla^{\prime} G_{i} d S^{\prime} d S \\
= \begin{cases}\iint_{S} \vec{f}_{m}(\vec{r}) \cdot \vec{E}^{\text {inc }} d S, & i=1 \\
0, & i=2,\end{cases}
\end{gathered}
$$

where subscript $i$ denotes the index of the medium. The details of the procedure can be found elsewhere (e.g., in $[25,26])$.

\section{Results and Discussion}

3.1. Human Eye Models. The first set of numerical results is obtained using the hybrid FEM/BEM formulation. The electric field induced in the extracted and the compound model of the eye, respectively, are given in Figures 4-7. The incident plane wave of $1 \mathrm{GHz}$ and $1800 \mathrm{MHz}$ is horizontally and vertically polarized and directed toward the corneal surface, perpendicular to the coronal head/eye cross-section. The amplitude of the incident EM wave is taken to be $1 \mathrm{~V} / \mathrm{m}$.

Figure 4 shows the results for the induced field on the surface of the extracted eye model and the compound eye model, respectively, due to incident wave of $1 \mathrm{GHz}$. From Figure 4 it is obvious that the distribution of electric field on the surface of the compound eye model (cornea and sclera) is not showing the symmetrical nature as is the case for the extracted eye model. In addition, the obtained peak values in the compound model are higher than the respected values in the extracted model, in addition to being different for both polarizations, again, contrary to the extracted model where the same values were obtained. These results suggest the significant influence of the neighboring head tissues on the induced electric field values.

The similar finding was shown for the $1800 \mathrm{MHz}$ case, as shown in Figure 5, although the discrepancy in the maximum values between the two models is significantly less.

It should also be noted that the extracted eye model gave highest values in the posterior parts denoting sclera, while the compound model obtained similar trend at both frequencies; that is, the highest obtained values are in the cornea.

More details on the distribution of the induced field inside the eye models can be seen in Figures 6 and 7 where the results for the induced field on the transverse cross-sections of the eye models and the electric field distribution along the eye visual axis, respectively, are shown.

The results from the previous study [8], obtained for vertically polarized wave at $1 \mathrm{GHz}$, showed similar distribution of the induced electric field along the pupillary axis in both compound and extracted eye models, as seen again in Figure 7(a), suggesting the usefulness of the extracted eye model in the initial EM exposure assessment. However, the results for horizontal polarization showed higher discrepancy. Moreover, as seen in Figure 7(b), this is even more pronounced at $1800 \mathrm{MHz}$, for both polarizations.

One interesting fact that can be seen from Figure 7 is that, due to the symmetrical nature of the employed eye model, the extracted model basically gives the same results for both 


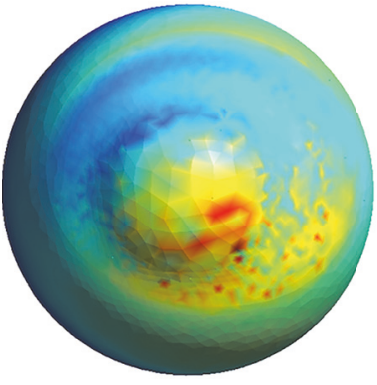

0.0124

(a)

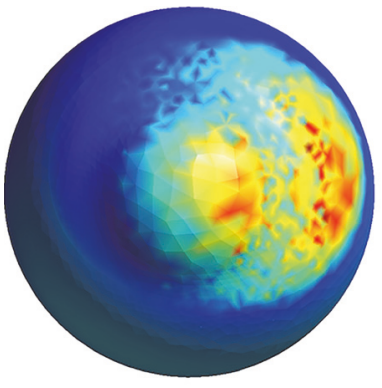

0.00256

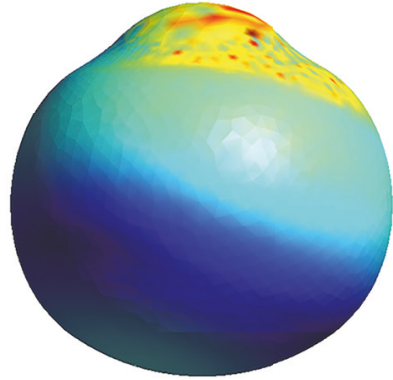

0.607

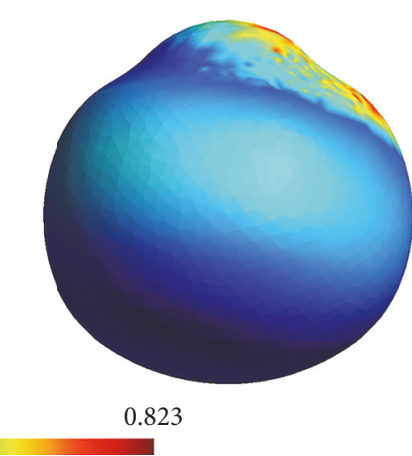

(c)

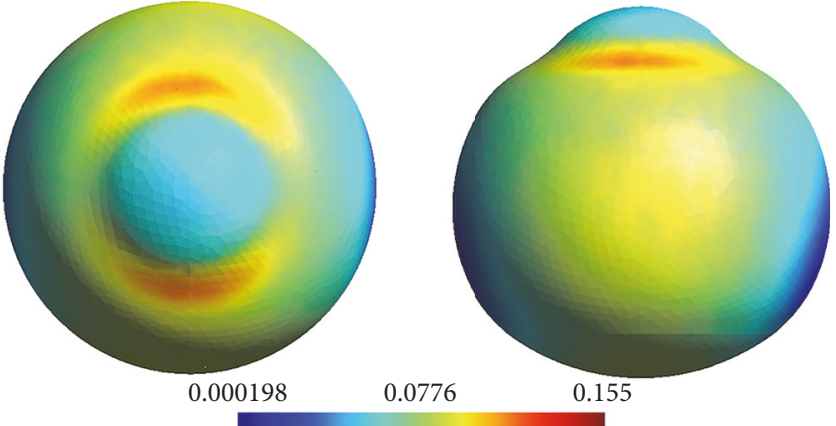

(b)

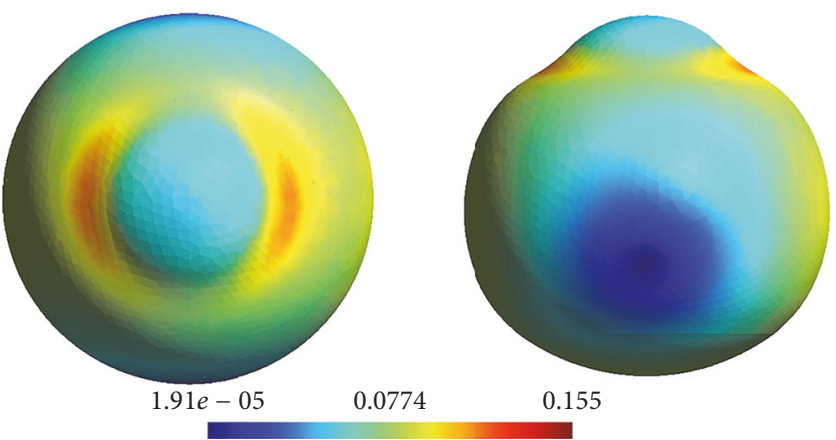

(d)

Figure 4: Induced electric field due to $1 \mathrm{GHz}$ EM wave on the surface of the eye (anterior and top view). (a, c) Compound eye model: (a) horizontal polarization and (c) vertical polarization. (b, d) Extracted eye model: (b) horizontal polarization and (d) vertical polarization.

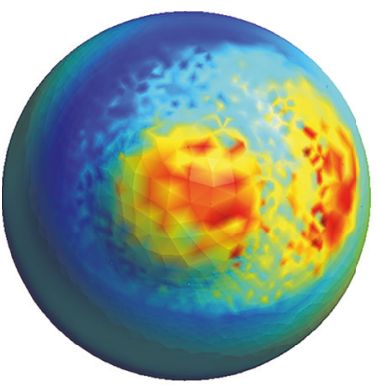

0.00264

264

0.238

(a)

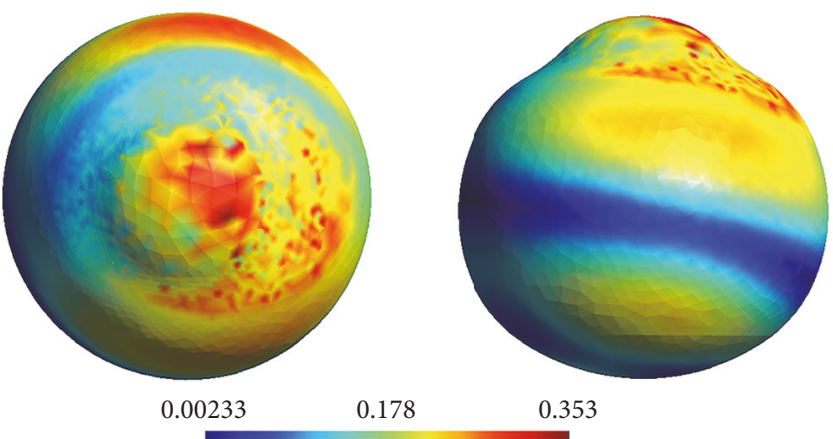

(c)

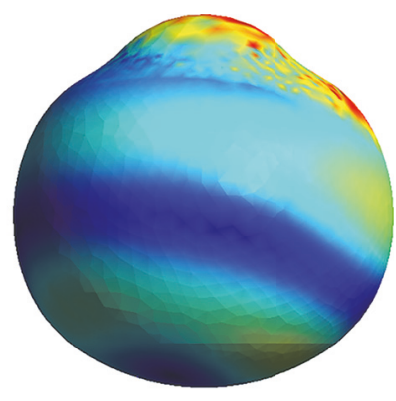

0.474

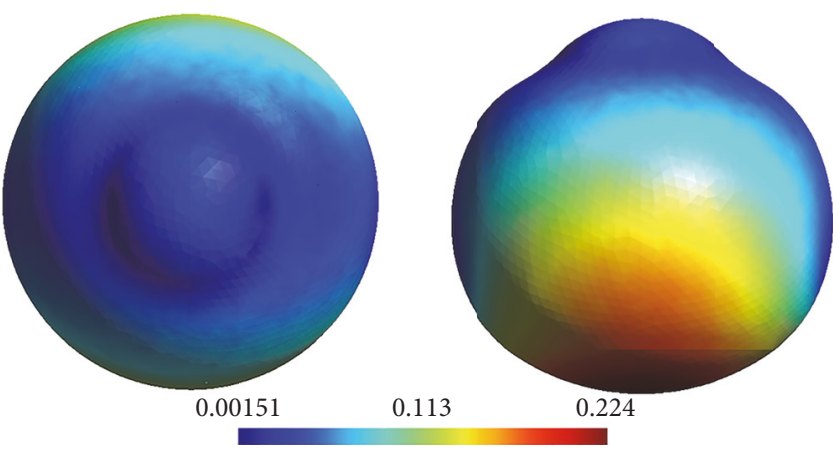

(b)
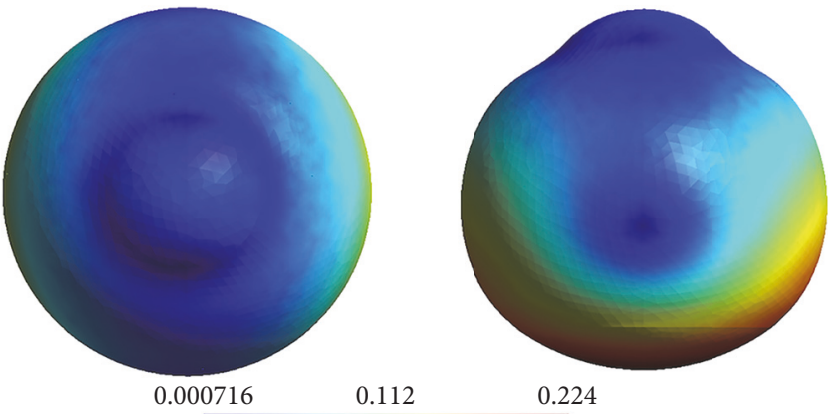

(d)

FigURE 5: Induced electric field due to $1800 \mathrm{MHz}$ EM wave on the surface of the eye (anterior and top view). (a, c) Compound eye model: (a) horizontal polarization and (c) vertical polarization. (b, d) Extracted eye model: (b) horizontal polarization and (d) vertical polarization. 


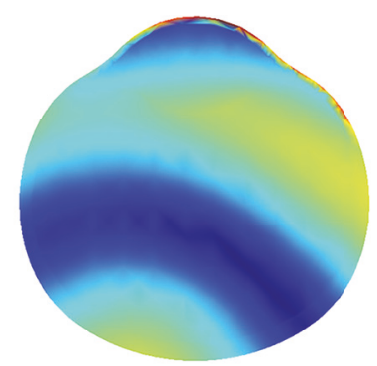

0.0321

0.217

0.402

(a)
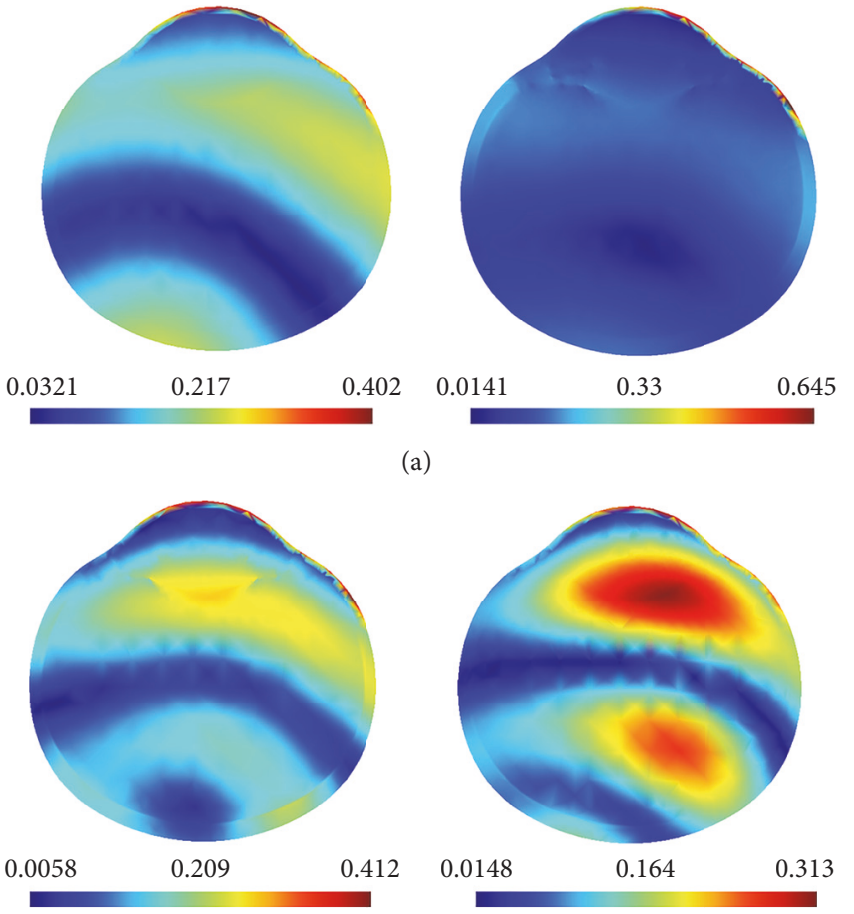

(c)

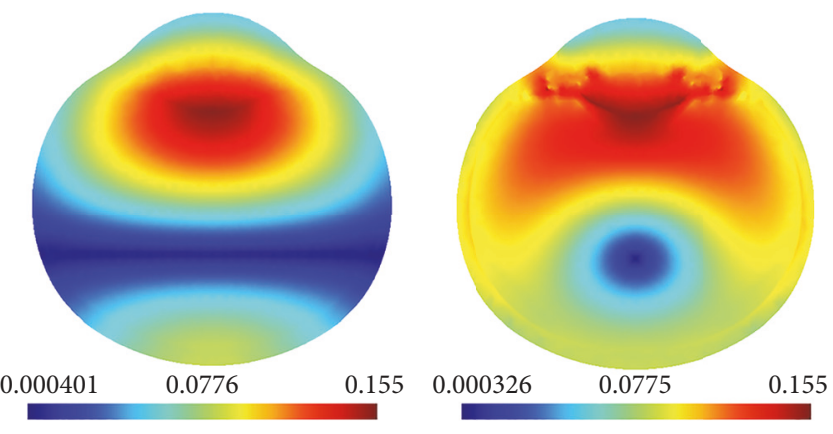

(b)

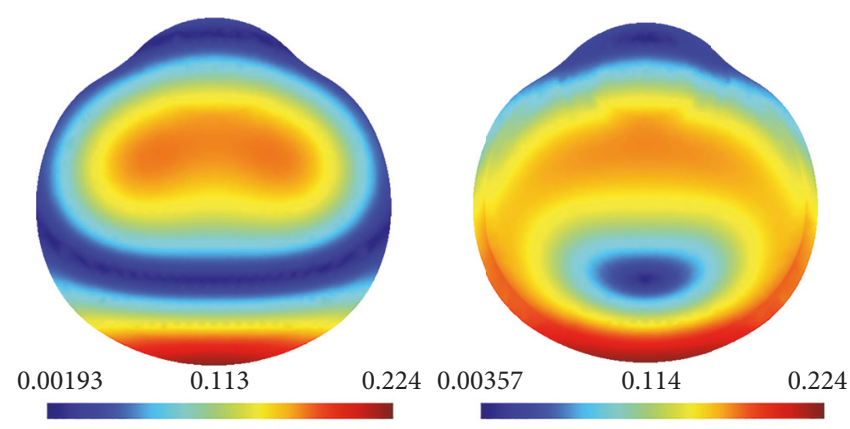

(d)

FiguRE 6: Induced electric field in the transverse cross-section of the compound eye model (a, c) and the extracted eye model (b, d). Incident EM wave of (a) and (b) $1 \mathrm{GHz}$ horizontal (left) and vertical (right) polarization and (c) and (d) $1800 \mathrm{MHz}$ horizontal (left) and vertical (right) polarization.

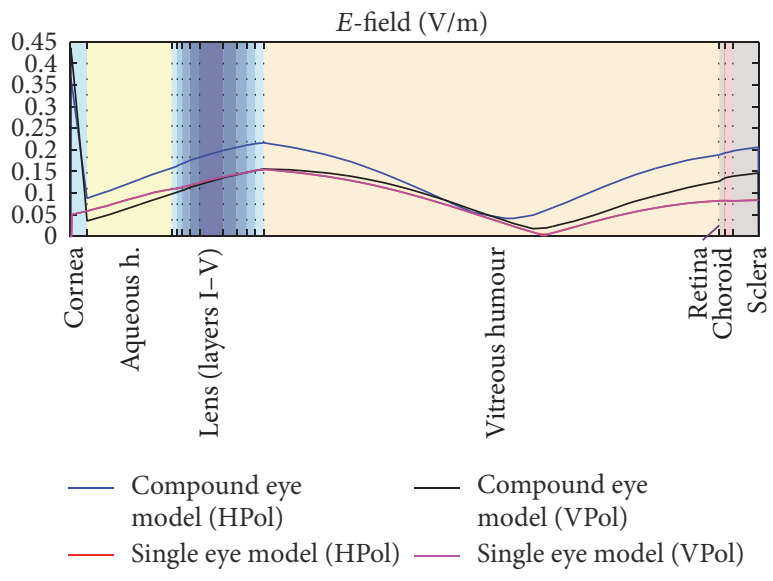

(a)

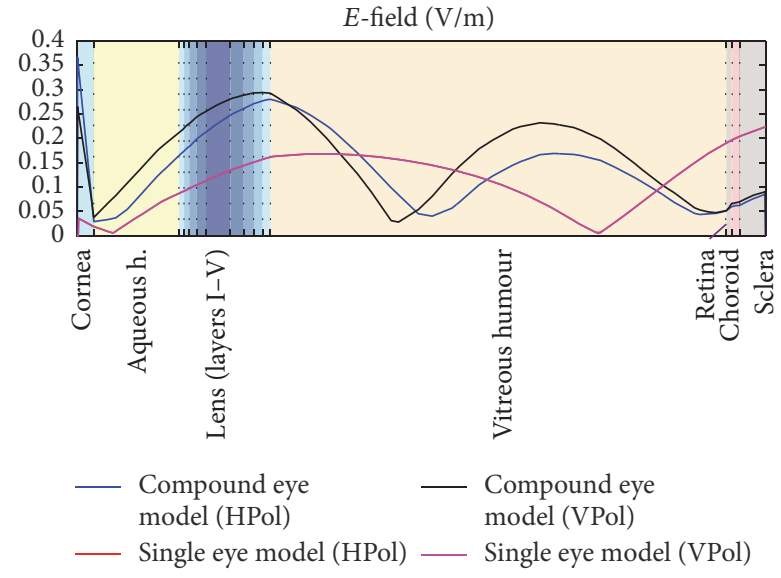

(b)

FIGURE 7: Comparison of the induced electric field along the pupillary axis of the compound and the extracted eye models, respectively, (a) $1 \mathrm{GHz}$, both polarizations; (b) $1800 \mathrm{MHz}$, both polarizations.

polarizations, as shown by almost ideally overlapping graphs, suggesting that this model does not discriminate between the two polarizations of the incident plane wave.

3.2. Human Brain Models. The following set of numerical results are obtained using the homogeneous brain model solved using the SIE/MoM formulation, while the results for the three-compartment model and the compound brain/head model are obtained using the hybrid FEM/BEM formulation.
The total of four different situations is considered, that is, that of both vertically and horizontally polarized plane wave of $900 \mathrm{MHz}$ and $1800 \mathrm{MHz}$ and amplitude of $1 \mathrm{~V} / \mathrm{m}$. The incident EM wave is directed toward the anterior part of the brain/head models.

The induced electric field on the brain surfaces of the three models is given in Figures 8-11. Although a similar distribution of the electric field was obtained, as shown by the colormaps, the lower maximum values were obtained in 

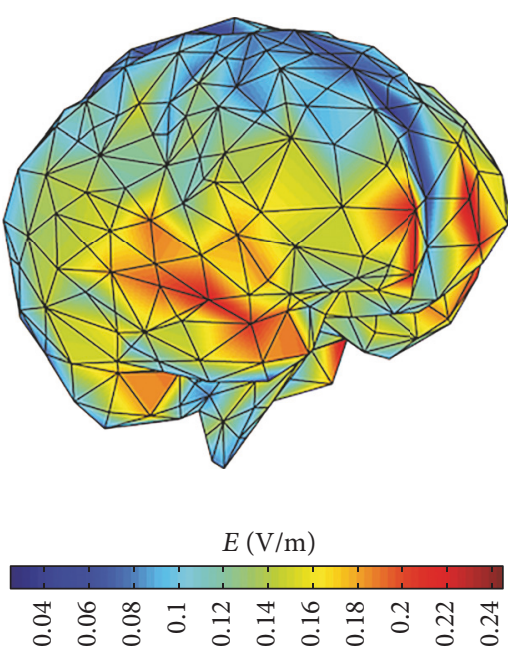

(a)

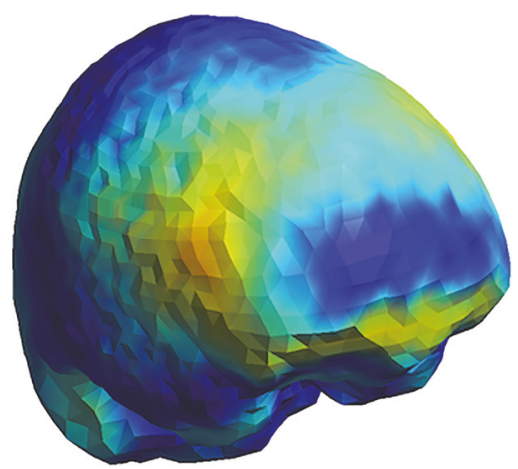

$E(\mathrm{~V} / \mathrm{m})$

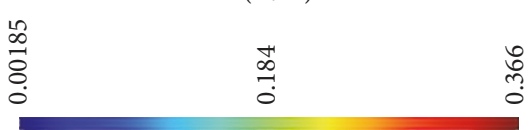

(b)

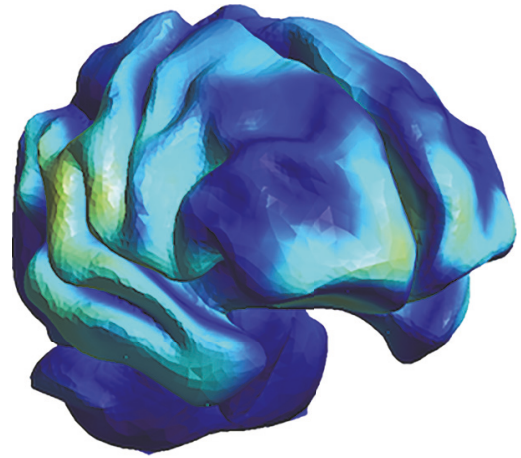

$E(\mathrm{~V} / \mathrm{m})$

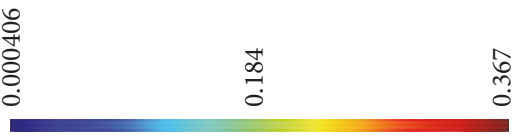

(c)

FiguRE 8: Induced electric field on the surface of the brain due to $900 \mathrm{MHz}$ horizontally polarized EM wave: (a) homogeneous model, (b) three-compartment model, and (c) compound model.
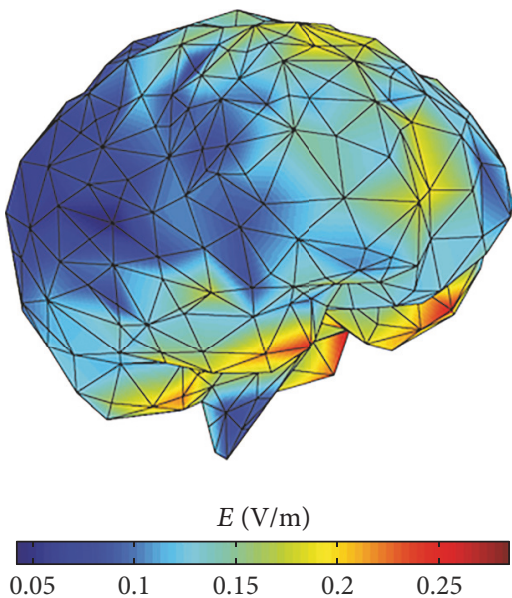

(a)

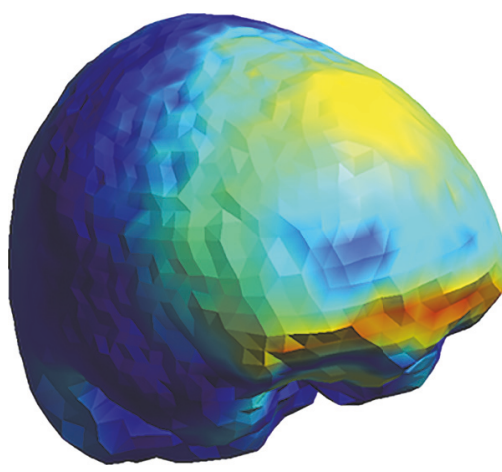

$E(\mathrm{~V} / \mathrm{m})$

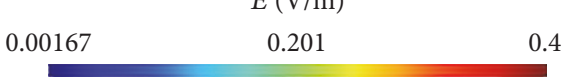

(b)

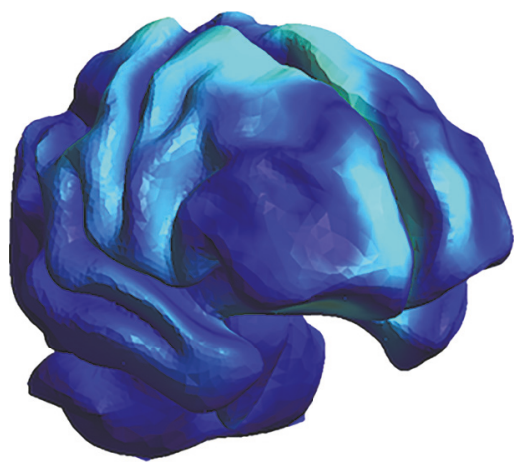

$E(\mathrm{~V} / \mathrm{m})$

$0.000624 \quad 0.234$

(c)

Figure 9: Induced electric field on the surface of the brain due to $900 \mathrm{MHz}$ vertically polarized EM wave: (a) homogeneous model, (b) three-compartment model, and (c) compound model.
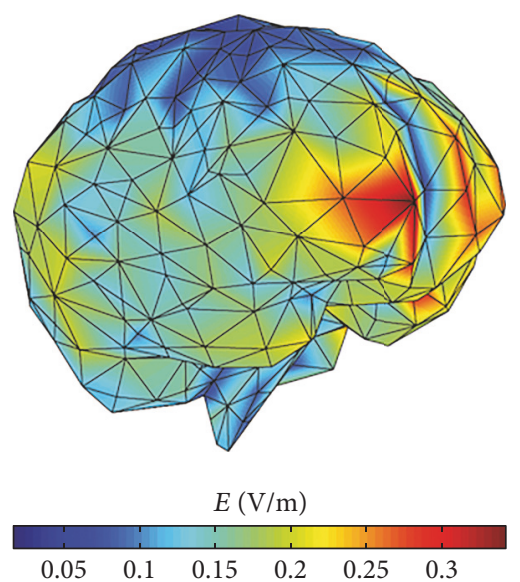

(a)

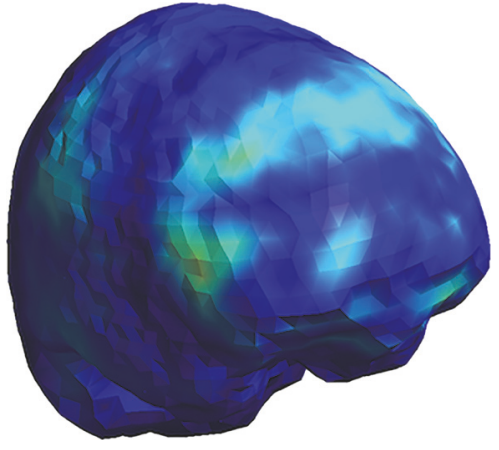

$E(\mathrm{~V} / \mathrm{m})$

0.000874

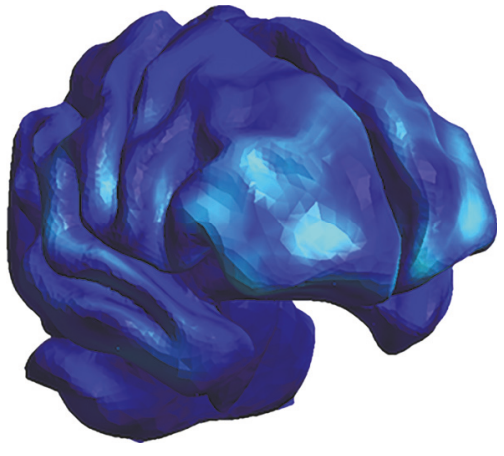

$E(\mathrm{~V} / \mathrm{m})$

$0.579 \quad 0.000177$ 0.463

(b)

FiguRE 10: Induced electric field on the surface of the brain due to $1800 \mathrm{MHz}$ horizontally polarized EM wave: (a) homogeneous model, (b) three-compartment model, and (c) compound model. 


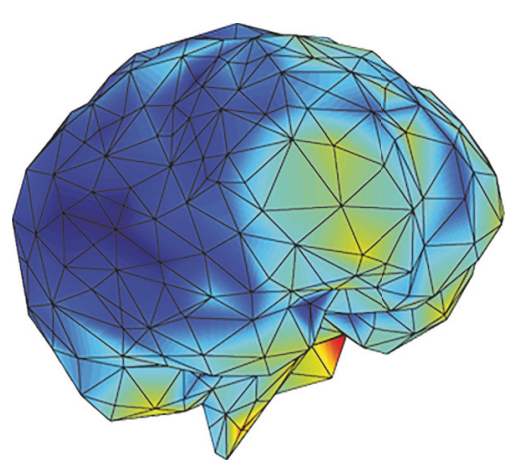

$E(\mathrm{~V} / \mathrm{m})$

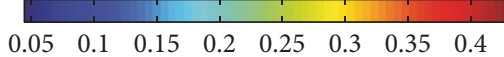

(a)

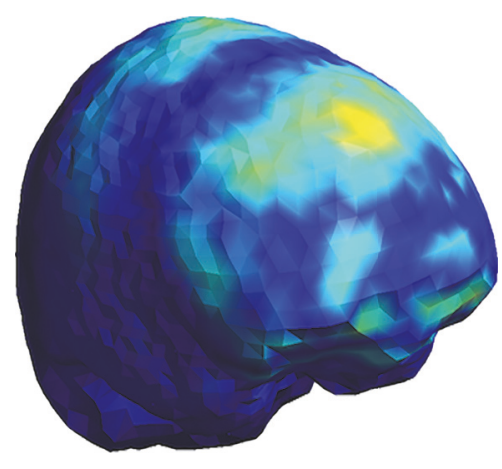

$E(\mathrm{~V} / \mathrm{m})$

0.253

(b)

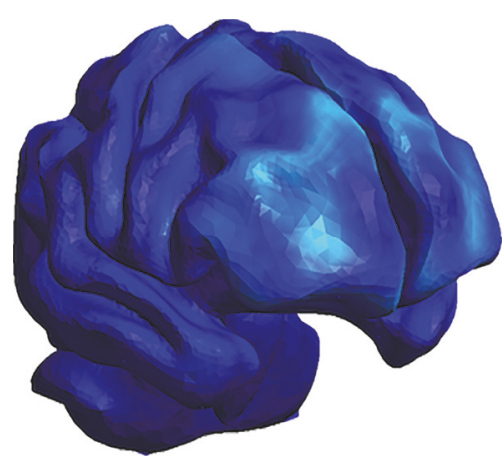

$E(\mathrm{~V} / \mathrm{m})$

0.268

(c)

Figure 11: Induced electric field on the surface of the brain due to $900 \mathrm{MHz}$ vertically polarized EM wave: (a) homogeneous model, (b) three-compartment model, and (c) compound model.

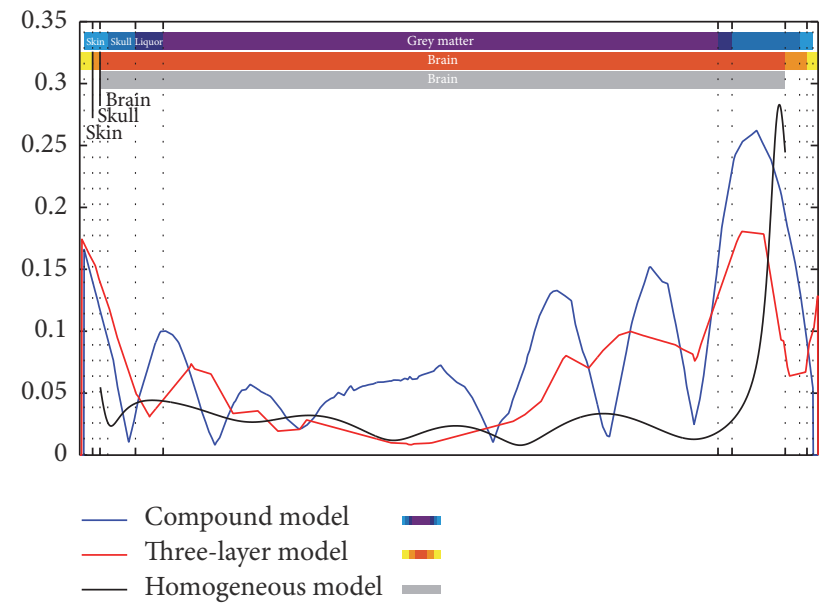

FIgURE 12: Comparison of the induced electric field along the sagittal axis of the homogeneous, the three-compartment, and the compound models, respectively, due to $900 \mathrm{MHz}$ EM wave, horizontal polarization, and incident on the anterior side.

all four cases in the homogeneous brain model compared to the other two brain models.

One drawback of the current implementation of the homogeneous model is a relatively low number of triangular elements used for the discretization of the brain surface; that is, the brain surface is tessellated using the $T=696$ triangular elements and $N=1044$ edge elements. The reason for such a low number is that employed SIE formulation results in fully populated matrices, thus preventing the current implementation from running on large system matrices. The low number of elements will also result in some numerical artifacts particularly evident in the superficial areas of the model, evident as peaks in Figures 12-15.

On the other hand, the results obtained using the two more elaborate models showed similar distributions for

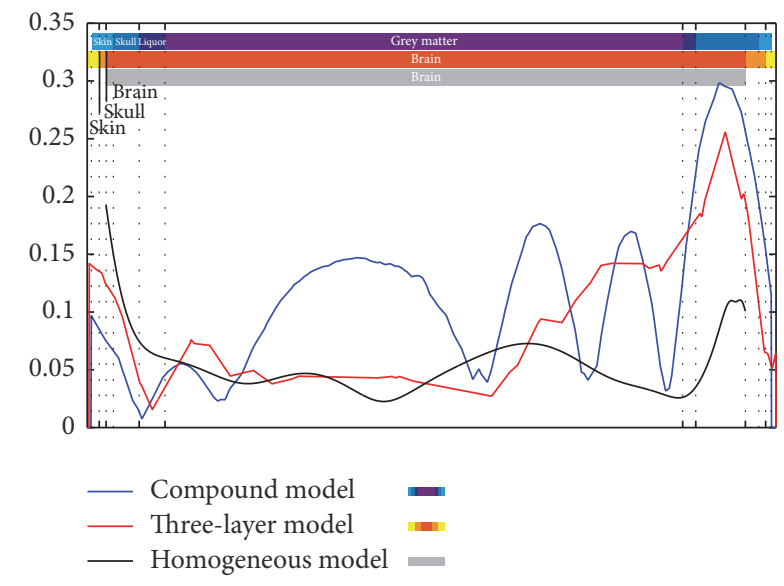

Figure 13: Comparison of the induced electric field along the sagittal axis of the homogeneous, the three-compartment, and the compound models, respectively, due to $900 \mathrm{MHz}$ EM wave, vertical polarization, and incident on the anterior side.

induced surface fields, as evident in Figures 8-11. Although the surface of three-compartment model is rather smoothed, lacking a cortex details featured in the conformed model, similar maximum values were obtained.

More details on the distribution of the electric field along the sagittal axis of the three brain models, obtained approximately at the medial prefrontal cortex, are seen in Figures 12-15. In general, similar trends are obtained in all models; however, only the detailed compound model shows the wavelike behavior in the interior domain.

The results from both $1800 \mathrm{MHz}$ cases showed that the three-compartment model obtained very uniform distribution of the field in the central part of the brain. In contrast to this, the homogeneous model showed field distribution in the central part of the brain much similar to the detailed compartment model. 


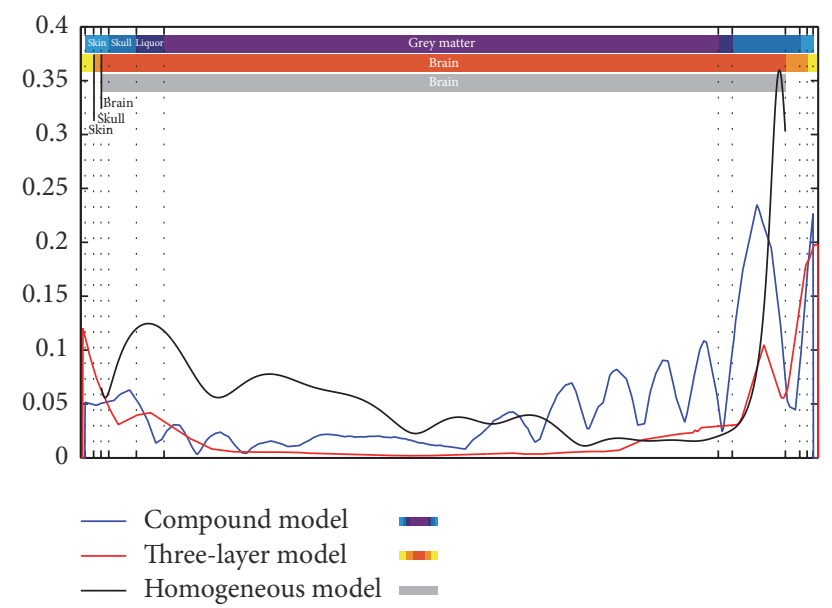

Figure 14: Comparison of the induced electric field along the sagittal axis of the homogeneous, the three-compartment, and the compound models, respectively, due to $1800 \mathrm{MHz}$ EM wave, horizontal polarization, and incident on the anterior side.

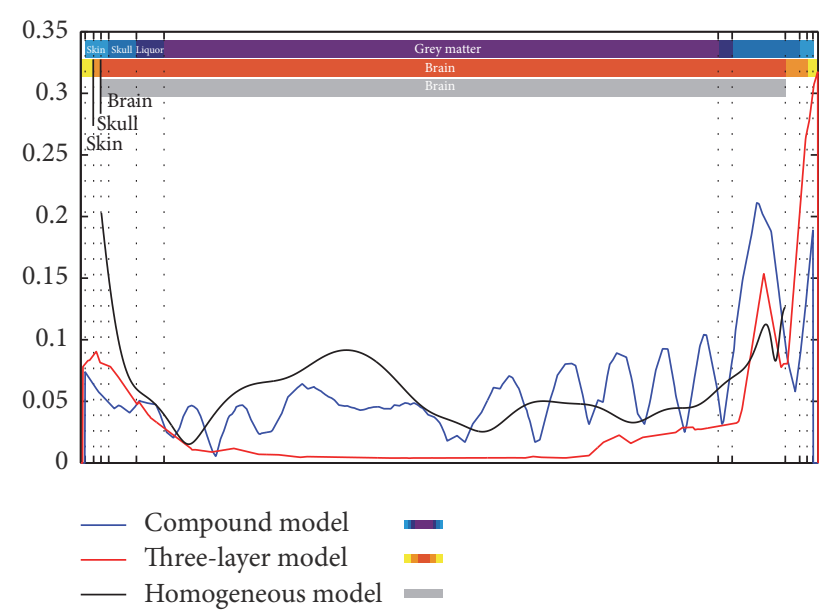

FIGURE 15: Comparison of the induced electric field along the sagittal axis of the homogeneous, the three-compartment, and the compound models, respectively, due to $1800 \mathrm{MHz}$ EM wave, vertical polarization, and incident on the anterior side.

\section{Conclusion}

The paper presented the comparison of the induced electric field in the extracted or the single organ models of the human eye and the brain, and the compound organ models incorporated in the detailed human head, respectively. The models of the human eye and brain were exposed to high frequency electromagnetic radiation of several frequencies and polarizations. The numerical results obtained using the hybrid FEM/BEM formulation showed similar distributions of the induced electric field along the pupillary axis in both the extracted and the compound eye models only in the case of $1 \mathrm{GHz}$ vertically polarized wave. However, the results for horizontal polarization and also for $1800 \mathrm{MHz}$ showed this not to be the case. Additionally, the study showed that extracted eye model due to its symmetrical nature obtained the similar results for both polarizations. The compound eye model showed different results, suggesting to be more appropriate when taking into account polarization of the incident electromagnetic wave.

The numerical results for the brain models showed similar maximum values and surface distributions for the three-compartment model and the compound model, while homogeneous model obtained significantly lower values. On the other hand, the homogeneous model obtained field distributions along the brain sagittal axis much similar to the compound model, while the three-compartment model at $1800 \mathrm{MHz}$ obtained somewhat unexpectedly a very uniform distribution in the brain tissue, dissimilar to the other two models.

\section{Conflicts of Interest}

The authors declare that they have no conflicts of interest.

\section{Acknowledgments}

The authors would like to thank I. Laakso from Aalto University, Finland, and A. Hirata from Nagoya Institute of Technology, Japan, for providing the detailed head model, and also A. Hunold and J. Haueisen from TU Ilmenau, Germany, for providing the three-compartment head model.

\section{References}

[1] J. W. Hand, "Modelling the interaction of electromagnetic fields $(10 \mathrm{MHz}-10 \mathrm{GHz}$ ) with the human body: Methods and applications," Physics in Medicine and Biology, vol. 53, no. 16, pp. R243-R286, 2008.

[2] D. Poljak, "Electromagnetic fields: Environmental exposure," in Encyclopedia of Environmental Health, Elsevier, 2011.

[3] D. Poljak, M. Cvetkovic, H. Dodig, and A. Peratta, "Electromagnetic-thermal analysis for human exposure to high frequency (HF) Radiation," International Journal of Design \& Nature and Ecodynamics, vol. 12, no. 1, pp. 55-67, 2017.

[4] K. D. Singh, N. S. Logan, and B. Gilmartin, “Three-dimensional modeling of the human eye based on magnetic resonance imaging," Investigative Ophthalmology \& Visual Science, vol. 47, no. 6, pp. 2272-2279, 2006.

[5] D. Poljak, Human Exposure to Electromagnetic Fields, WIT Press, Southampton-Boston, 2003.

[6] M. J. Ackerman, "The visible human project," Proceedings of the IEEE, vol. 86, no. 3, pp. 504-511, 1998.

[7] J. W. Massey and A. E. Yilmaz, "AustinMan and AustinWoman: High-fidelity, anatomical voxel models developed from the VHP color images," in Proceedings of the 38th Annual International Conference of the IEEE Engineering in Medicine and Biology Society, EMBC 2016, pp. 3346-3349, USA, August 2016.

[8] M. Cvetkovic', H. Dodig, and D. Poljak, "Comparison of Numerical Electric Field and SAR Results in Compound and Extracted Eye Models," in Proceedings of the 2nd International Multidisciplinary Conference on Computer and Energy Science (SpliTech), pp. 1-6, Split, Croatia, 2017.

[9] H. Dodig, D. Poljak, and A. Peratta, "Hybrid BEM/FEM edge element computation of the thermal rise in the $3 \mathrm{D}$ model of the human eye induced by high frequency em waves," in Proceedings of the 2012 20th International Conference on Software, 
Telecommunications and Computer Networks, SoftCOM 2012, hrv, September 2012.

[10] M. Cvetković and D. Poljak, "An efficient integral equation based dosimetry model of the human brain," in Proceedings of the 2014 International Symposium on Electromagnetic Compatibility, EMC Europe 2014, pp. 375-380, Sweden, September 2014.

[11] B. U. Forstmann, M. C. Keuken, A. Schafer, P.-L. Bazin, A. Alkemade, and R. Turner, "Multi-modal ultra-high resolution structural 7-Tesla MRI data repository," Scientific Data, vol. 1, Article ID 140050, 2014.

[12] M. C. Keuken, P.-L. Bazin, A. Schäfer, J. Neumann, R. Turner, and B. U. Forstmann, "Ultra-high 7T MRI of structural agerelated changes of the subthalamic nucleus," The Journal of Neuroscience, vol. 33, no. 11, pp. 4896-4900, 2013.

[13] J. Budde, G. Shajan, K. Scheffler, and R. Pohmann, "Ultra-high resolution imaging of the human brain using acquisitionweighted imaging at 9.4T,' NeuroImage, vol. 86, pp. 592-598, 2014.

[14] C. Gabriel, "Compilation of the Dielectric Properties of Body Tissues at RF and Microwave Frequencies.," Defense Technical Information Center, 1996.

[15] A. Gullstrand, "The optical system of the eye," Physiological Optics, vol. 1, pp. 350-358, 1909.

[16] J. A. Díaz, C. Pizarro, and J. Arasa, "Single dispersive gradientindex profile for the aging human lens," Journal of the Optical Society of America A: Optics and Image Science, and Vision, vol. 25, no. 1, pp. 250-259, 2008.

[17] I. Laakso, S. Tanaka, S. Koyama, V. De Santis, and A. Hirata, "Inter-subject variability in electric fields of motor cortical tDCS," Brain Stimulation, vol. 8, no. 5, pp. 906-913, 2015.

[18] S. M. Blinkov and I. I. Glezer, The Human Brain in Figures and Tables. A Quantitative Handbook, Plenum Press, New York, USA, 1968.

[19] D. Poljak, M. Cvetkovic, O. Bottauscio et al., "On the Use of Conformal Models and Methods in Dosimetry for Nonuniform Field Exposure," IEEE Transactions on Electromagnetic Compatibility, pp. 1-10.

[20] M. Stenroos, A. Hunold, and J. Haueisen, "Comparison of threeshell and simplified volume conductor models in magnetoencephalography," NeuroImage, vol. 94, pp. 337-348, 2014.

[21] D. Poljak, D. Cavka, H. Dodig, C. Peratta, and A. Peratta, "On the use of the boundary element analysis in bioelectromagnetics," Engineering Analysis with Boundary Elements, vol. 49, pp. 2-14, 2014.

[22] J.-C. Nédélec, "Mixed finite elements in R3," Numerische Mathematik, vol. 35, no. 3, pp. 315-341, 1980.

[23] J. L. Volakis, A. Chatterjee, and L. C. Kempel, Finite Element Method Electromagnetics: Antennas, Microwave Circuits, and Scattering Applications, vol. 6, John Wiley \& Sons, 1998.

[24] H. Dodig, EM waves and the human eye [Ph.D. thesis], 2012, Wessex Insitute of Technology, available NLW South ARCHIVES (2013/0029), National Library of Wales MMS ID:99205473102419.

[25] D. Poljak, M. Cvetkovic', A. Peratta, C. Peratta, H. Dodig, and A. Hirata, "On some integral approaches in electromagnetic dosimetry," in BioEM, pp. 289-296, Ghent, Belgium, 2016.

[26] M. Cvetković, D. Poljak, and J. Haueisen, "Analysis of transcranial magnetic stimulation based on the surface integral equation formulation," IEEE Transactions on Biomedical Engineering, vol. 62, no. 6, pp. 1535-1545, 2015. 


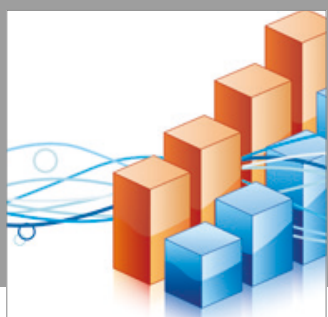

Advances in

Operations Research

vatersals

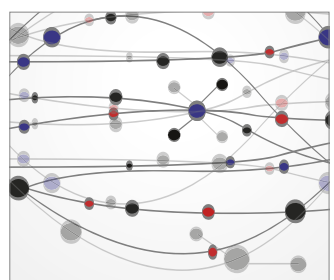

\section{The Scientific} World Journal
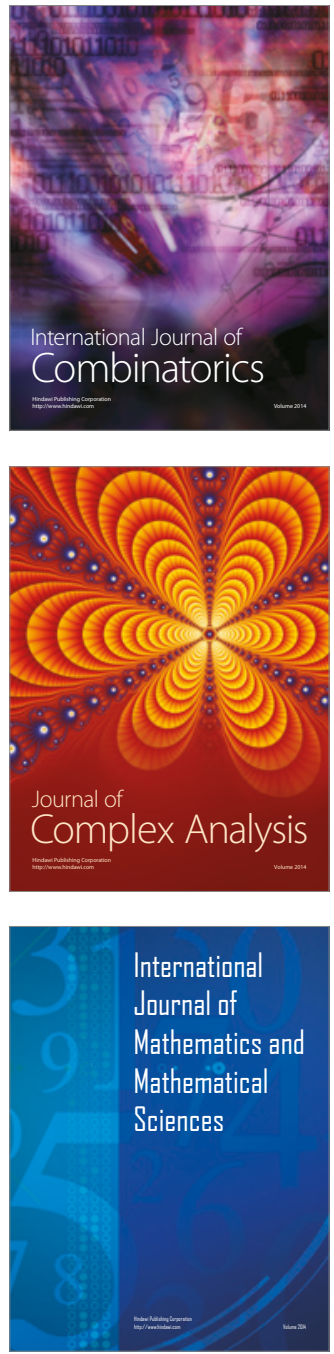
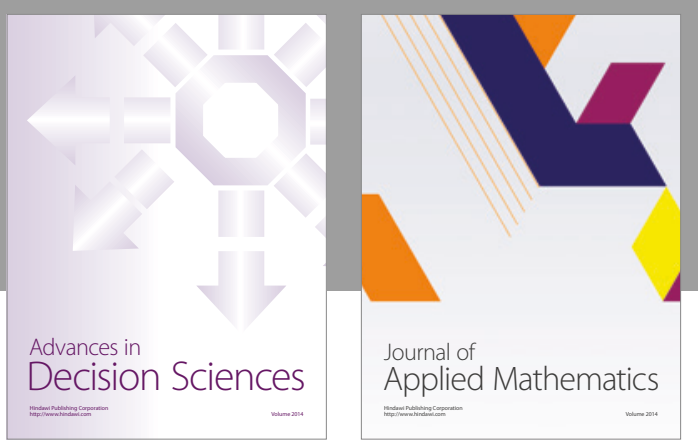

Algebra

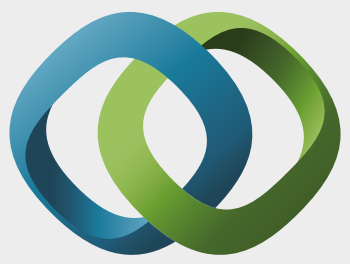

\section{Hindawi}

Submit your manuscripts at

https://www.hindawi.com
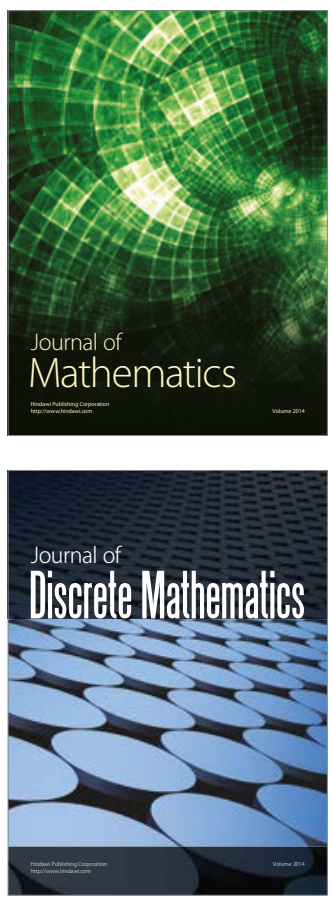

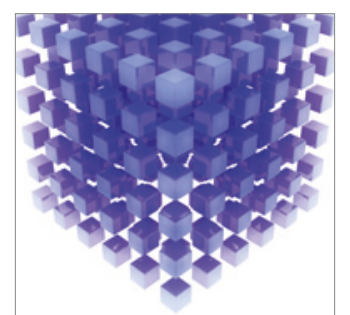

Mathematical Problems in Engineering
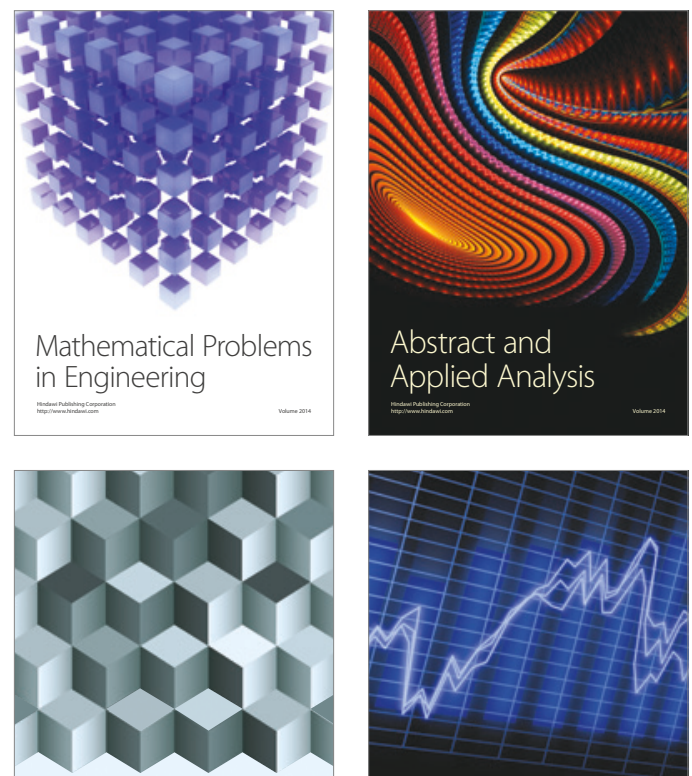

Journal of

Function Spaces

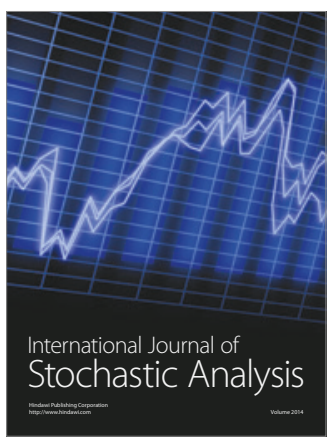

Probability and Statistics
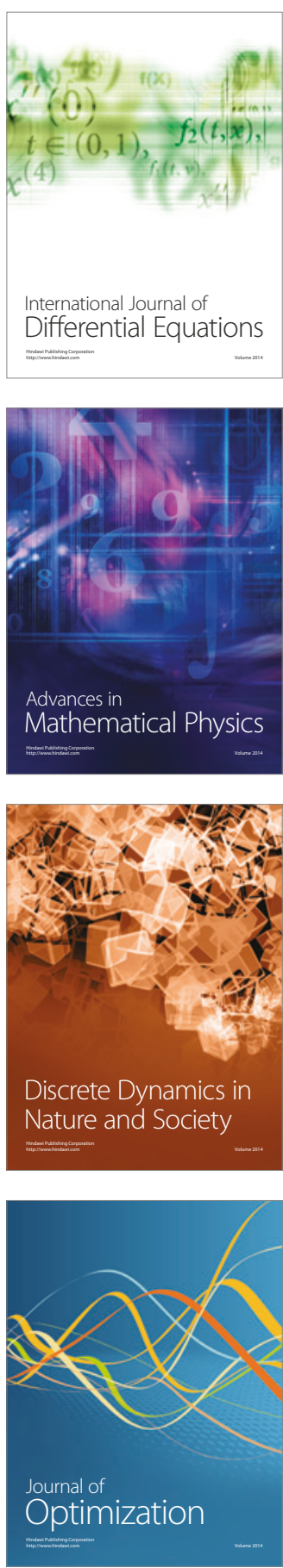